\title{
Risk loci, biological candidates and biomarkers
}

Three genome-wide association studies of prostate cancer deliver a mix of newly identified associations, tripling the
available number of candidate genes, biomarkers and variants potentially useful in risk prediction.

A number of recent studies published in the journal have established robust associations of common variants at five genetic loci with prostate cancer, a common disease for which we had very few prior clues to its familial predisposition or even to its biology (Nat. Genet. 38, 652-658; 2006, 39, 631-637; 2007, 39, 638-644; 2007, 39, 645-649; 2007, 39 954-956; 2007, 39, 977-983; 2007, 39, 989-994; 2007, News and Views 39, 579-580; 2007, Editorial 39, 569; 2007).

Now three genome-wide association studies by Julius Gudmundsson and colleagues, Gilles Thomas and colleagues and Rosalind Eeles and colleagues (pp 281, 310, and 316) confirm those loci and report replicated, highly significant associations with common SNPs at another ten newly identified loci.

Two of the three studies identified associated SNPs at chromosome 10 near the $M S M B$ gene. Thomas et al. note that not only do the associated promoter variants affect expression of this gene in vitro, but serum expression measurements of the gene's seminal MSP protein have been used for early detection of high-grade disease and expression is lost in advanced androgen-insensitive prostate cancer. So, they suggest that this is a locus for which the study has delivered a genetic risk marker, improved our understanding of a biomarker and supplied evidence for functional involvement in disease progression. Risk variants at Xp11.22 were also found by two of the three studies.

A currently used biomarker for prostate cancer diagnosis is an elevated serum concentration of PSA, a prostatic protease. Whatever its value in individual clinical decisions, PSA has played two roles in this genetic epidemiology. First, Eeles et al. suggest that their success may have hinged upon the choice of controls with particularly low PSA levels in their screen, together with a primary case population enriched for early onset or family history of prostate cancer. Second, one of the significantly associated SNP markers falls between the KLK3 and KLK2 genes - the first encoding PSA itself, and the latter a similar enzyme that may also be a useful biomarker.

One of the mysteries of prostate cancer is that most men affected will die with-rather than from-the disease. It is therefore important to distinguish cancers with different courses and genetic background may play a role. Thomas et al. provide free access to the computed statistics of the genotype counts for each SNP they tested by phenotypic class: control, aggressive cancer, and nonaggressive cancer. Gudmundsson et al. found that the $2 \mathrm{p} 15$ variant was significantly associated with aggressive as compared to nonaggressive disease.

Thomas et al. emphasize that selecting a large number of markers for replication is essential if the method is to have a chance of delivering a significant association, noting that two of the markers that eventually produced a replicated positive were ranked far from the top of their initial hits (to make the top 5\% in a GWAS is to be jockeying for position among scores of thousands of likely markers). The sparse overlap of the loci significantly associated in the three studies, and the additional nine suggestive associations reported by Thomas et al. show that sharing of results and collaborative analysis of all the available studies is likely to be productive. The three groups used slightly different variations of a very similar experimental design, which suggests that more common loci associated with prostate cancer are yet to be discovered. 